\title{
Influence of carbon nanofibers electric conductivity on infrared extinction capability
}

\author{
Tao SHEN ${ }^{1, a}$, Wei Jun YANG, Bai He WANG, Min Li YAO \\ ${ }^{1}$ Xi'an Research Institute of High Technology, Shannxi, China
}

\begin{abstract}
Using method of moment, the calculating model of electric current distributing and scattered electric field for carbon nanofibers is established in this paper. Based on it, the expression of absorption cross section, scattering cross section and extinction cross section are got. The relationship between infrared extinction cross section of carbon nanofiber and electrical conductivity is analyzed and calculated by using Matlab. The results show that the infrared extinction cross section increases with electrical conductivity increase and peak value appeares when wavelength is longer than the length of carbon nanofiber. The electrical conductivity on peak value is related to wavelength.
\end{abstract}

Keywords- carbon nanofibers; infrared extinction; absorption; scattering; extinction coefficient

\section{INTRODUCTION}

Smoke is a simple and effectual means of laser passive interference, and the infrared smoke materials should be taken on the better infrared extinction capability. Carbon nanofiber is a new kind of materials with good capability of electromagnetic wave absorption. It not only has the characteristic of common gas phase growth carbon fiber, but also has the characteristic of low density, high electrical conductivity, large specific surface and high specific intensity. In infrared band, the length and wavelength of carbon nanofiber is the same order. It can generate not only scattering attenuation, but also absorption attenuation. In modern warfare, carbon nanofiber has been a new kind of infrared smoke interference materials. To explore the applications of carbon nanofibers in passive interference by smoke screen, the integral equation of induced cueernt for carbon nanofibers is established based the electromagnetic field theory.

\section{The integral equation for carbon nanofiber}

To carbon nanofiber, the radius is much shorter than the length and incidence wavelength. The induced current inside carbon nanofiber is uniform distribution along the axisl direction and no component is in radial direction. According to the axial symmetry, the axisl direction current can replace the total induced current I. Suppose the length direction as $\mathrm{Z}$ axis, the scattered field produced by the axisl direction current in $r$ point is following:

$$
\begin{aligned}
& E(r)=-j w u \int\left[1+\frac{1}{k^{2}} \nabla^{2}\right] I\left(z^{\prime}\right) \times G\left(r, z^{\prime}\right) d z^{\prime} \\
& G\left(r, z^{\prime}\right)=\frac{\exp \left(-j k\left|r-z^{\prime}\right|\right)}{4 \pi\left|r-z^{\prime}\right|} \text { is Green function. }
\end{aligned}
$$

The carbon nanofiber produces the conduction current by field: $I_{1}=\pi a^{2} \sigma E_{n e i}, E_{n e i}$ is the internal field, $\sigma$ is carbon nanofiber conductivity; the dielectric property of the materials can also produce polarization current[1] $I_{2}=j \pi a^{2} \omega\left(\varepsilon-\varepsilon_{0}\right) E_{n e i}, \varepsilon$ is the dielectric constant, then the total induced current is:

$$
I(z)=I_{1}+I_{2}=\pi a^{2}\left[\sigma+j \omega\left(\varepsilon-\varepsilon_{0}\right)\right] E_{\text {nei }}
$$

The relationship between the induced current and the internal field of the carbon nanofiber is:

$$
E_{n e i}=\frac{I(z)}{\pi a^{2}\left[\sigma+j \omega\left(\varepsilon-\varepsilon_{0}\right)\right]}
$$

The field and internal field of the materials is the same along the $\mathrm{Z}$ axis. The field is composed by incidence field Ei and scattered field Es and the boundary condition is

$$
E_{n e i}=E_{i z}+E_{s z} \text { surface }
$$

(1)and(3) is substituted in (4)

$$
\begin{aligned}
& \tau \mathrm{g} J(z)+j \omega \mu \int\left(1+\frac{1}{k^{2}} \frac{\partial^{2}}{\partial z^{2}}\right) I\left(z^{\prime}\right) G\left(z, z^{\prime}\right) d z^{\prime}=E_{i z} \\
& \tau=\frac{1}{\pi a^{2}\left[\sigma+j \omega\left(\varepsilon-\varepsilon_{0}\right)\right]} \text { Equation(5) is the }
\end{aligned}
$$

Equation(5) is the carbon nanofiber integral equation. The method of moment(MOM) is used to solve the integral equation.

\footnotetext{
a Corresponding author: luckyshentao@163.com
} 
First, dividing L length carbon nanofiber into homogeneous $\mathrm{N}$ segment, each segment length is $\Delta l=L / N$, the whole integrating range is composed by $\mathrm{N}$ sub-interval.

$$
I=\sum_{n=1}^{N} I_{n} P_{n} \quad n=1,2, \mathrm{~K} N
$$

$P_{n}=\left\{\begin{array}{lc}1 & \text { inLn } \\ 0 & \text { others }\end{array}\right.$

(6) is substituted in (5)

$$
\tau \mathrm{g} \sum_{n=1}^{N} I_{n} P_{n}+j \omega \mu \sum_{n=1}^{N} I_{n} \times \int_{L n}\left[1+\frac{1}{k^{2}} \frac{\partial^{2}}{\partial z^{2}}\right] G\left(z, z^{\prime}\right) d z^{\prime}=E_{i z}
$$

Select point matching weight function $\omega_{m}=\delta\left(z-z_{m}\right)$ to multiply formula(7),

$$
\tau \mathrm{g} \sum_{n=1}^{N} I_{n} \delta_{m n}+j \omega \mu \sum_{n=1}^{N} I_{n} \times \int_{L n}\left[1+\frac{1}{k^{2}} \frac{\partial^{2}}{\partial z_{m}{ }^{2}}\right] G\left(z_{m}, z^{\prime}\right) d z^{\prime}=E_{i z}\left(z_{m}\right)
$$

The induced current distribution inside the carbon nanofiber can be obtained by solving the matrix equation above.

\section{3 carbon nanofiber extinction cross section}

In far field condition, According to formula (1), the carbon nanofiber scattered field is

$E_{s}(r)=\frac{j \omega \mu_{0}}{4 \pi} \frac{\exp (-j k r)}{r} \sin \theta \hat{\mathrm{g}} \times \int_{-L / 2}^{L / 2} I\left(z^{\prime}\right) \exp \left(j k z^{\prime} \cos \theta\right) d z^{\prime}$

(6) is substituted in (10)

$E_{s}(r)=\hat{\theta} \frac{\eta_{0}}{4 \pi} \frac{\exp (-j k r)}{r} \operatorname{tg} \theta \mathrm{g} \times \sum_{n-1}^{N} I_{n}\{\exp (j k n \Delta l \cos \theta)-\exp [j k(n-1) \Delta l \cos \theta]\}$

$\eta_{0}=\left(\mu_{0} / \varepsilon_{0}\right)^{1 / 2}$

To unit amplitude incidence wave, the scattered field can be obtained from above and the carbon nanofiber scattering cross section is

$\sigma_{s}=\frac{1}{I_{0}} \int I_{s} d s=2 \pi \int_{0}^{\pi}\left|E_{s}\right|^{2} r^{2} \sin \theta d \theta$

the carbon nanofiber absorption cross section is

$\sigma_{a}=\int_{V} k_{0} \varepsilon_{r}{ }^{\prime \prime}\left|E_{n e i}\right|^{2} d V=\frac{\sigma \sqrt{\frac{\mu_{0}}{\varepsilon_{0}}}}{\pi a^{2}\left|\sigma+j \omega\left(\varepsilon-\varepsilon_{0}\right)\right|^{2}} \sum_{i=1}^{N}\left|I_{n}\right|^{2} \Delta l$

extinction cross section is composed by absorption cross section and scattering cross section, that is

$$
\sigma_{e}=\sigma_{a}+\sigma_{s}
$$

\section{Calculation and discussion}

The code of carbon nanofiber induced current, scattered field and extinction cross section are programmed based on Matlab. The relationship between infrared extinction cross section of carbon nanofiber and electrical conductivity is analyzed and calculated. The calculation conditions are:

$$
\varepsilon_{r}=10, a=50 \mathrm{~nm}, L=5 \mu \mathrm{m}, N=50
$$

The calculation result is as followed Fig.1.
The results show that when the wavelength is $2 \mu \mathrm{m}$, extinction cross section increases fast with electrical conductivity increase.

When $\sigma<6 \times 10^{5} \Omega^{-1} \mathrm{~m}^{-1}$, carbon nanofiber absorbs the incidence wave more than the scattered wave. When $\sigma>6 \times 10^{5} \Omega^{-1} \mathrm{~m}^{-1}$, extinction effect is mainly the scattering effect, absorbing effect is weak. When the wavelength is $5 \mu \mathrm{m}$, the extinction cross section peak value appeares near the electrical conductivity $\sigma=10^{6} \Omega^{-1} \mathrm{~m}^{-1}$. The absorbing effect is tapered with electrical conductivity increasing, the scattering effect is enhanced and the extinction cross section reduces little. When the electrical conductivity is near $10^{7} \Omega^{-1} m^{-1}$, the extinction cross section keeps constant. When incidence wavelength is $14 \mu \mathrm{m}$ and $20 \mu \mathrm{m}$, the extinction cross section has peak value. It is $3 \times 10^{6} \Omega^{-1} \mathrm{~m}^{-1}$ and $8 \times 10^{5} \Omega^{-1} m^{-1}$ accordingly. To $14 \mu \mathrm{m}$ wavelength, when $\sigma<7.8 \times 10^{6} \Omega^{-1} m^{-1}$, the extinction effect mainly is absorbing effect. To $20 \mu \mathrm{m}$ wavelength, when $\sigma<2 \times 10^{7} \Omega^{-1} \mathrm{~m}^{-1}$, the extinction effect mainly is scattering effect. So, the longer the wavelength is, the larger the electrical conductivity span of absorbing extinction is.

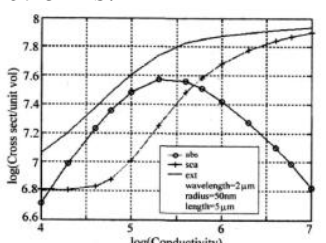

(a)

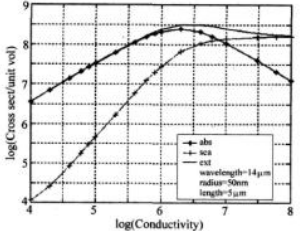

(c)

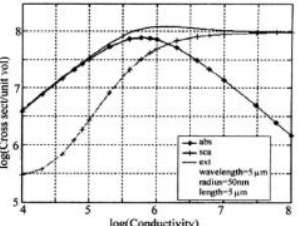

(b)

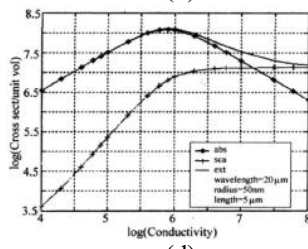

(d)
Fig.1 Relationship between electrical conductivity and extinction cross section

\section{Conclusion}

According to the electrical conduction and dielectric characters of carbon nanofiber, the electromagnetic field integral equation is built. The integral equation is solved and the expression of absorption cross section, scattering cross section and extinction cross section are got. The relationship between infrared extinction cross section of carbon nanofiber and electrical conductivity is analyzed and calculated based on Matlab. The results show that the infrared extinction cross section increases with electrical conductivity increase and peak value appeares when wavelength is longer than the length of carbon nanofiber. The electrical conductivity on peak value is related to wavelength. The analysis results can supply the theoretical basis for IR smoke materials in preparation and selection.

\section{ACKNOWLEDGEMENTS}

This work was in part supported by China Postdoctoral Science Foundation (2015M572698) and 
Shaanxi Technology Committee Natural Science Basic Research Project(2014JM2-6108).

\section{References}

[1] DU Jin hong, SUN Chao, BAI Shuo, et a1. Microwave electromagnetic characteristics of a microcoiled carbon fibers/paraff in wax composite in $\mathrm{Ku}$ band[J] . J Mater Res, 2002, 17( 5) : 1232-1236

[2] Lee B O, Woo W J, Park H S, et al. Infulence of aspect ratio and skin effect on EMI shielding of coating materials fabricated with carbon nanofiber/ PVDF[J]. J Mater Sci, 2002, 37: 1839.

[3] Xiao Chun Nie, Le Wei Li, Ning Yuan, et al. A fast analysis of electromagnetic scattering by arbitrarily shaped homogeneous dielectric object s[J] . Micro Opt Tech Lett, 2003, 38( 1) : 30-35.

[4] Deitzel ,J M, Kleinmeyer ,J, Harris D, et al. The effect of processing variables on the morphology of electrospun nanofibers and textiles [J]. Polymer, 2011, 42(1): 261-272.

[5] Jun Kameoka, Reid Orth, Yanou Yang, et al. A scanning tip electrospinning source for deposition of oriented nanofibres[J]. Nanotechnology, 2003, 14(10): 1124-1129.

[6] Zhang Zhenhua, Peng Jingcui. The electronic structure and magnetic properties of chiral toroidal carbon nanotubes $[\mathrm{J}]$. Act a Physica Sinica, 2011, 50(6) : 1150 . 